\title{
Influence of finite notch root radius and optically determined crack length on the measured fracture toughness of brittle materials
}

\author{
Hüseyin Özcoban, Hans Jelitto, Gerold A. Schneider \\ Institute of Advanced Ceramics, \\ Hamburg University of Technology, Hamburg, Germany
}

\begin{abstract}
When measuring the fracture toughness of ceramics, for instance with the Single Edge VNotched Beam (SEVNB) method, a known problem is given by the finite notch root radius. If this radius is larger than a certain small value of a few micrometers, the measured stress intensity factor is higher than the true intrinsic value. Another problem is that due to technical and physical reasons the optically measured crack length at stable crack growth is smaller than the true crack length, which might lead to an underestimation of the fracture toughness. In the given paper an experimental approach is presented to register these effects quantitatively with stable crack advance and to finally obtain the correct fracture toughness. This is achieved by applying a new computer aided automatic control system for stable crack advance and by analyzing the results in force - displacement and crack length - force diagrams. The used material is zirconia-toughened alumina.
\end{abstract}

\section{Introduction}

Ceramic materials fail in a brittle manner due to stress concentration at crack tips. Therefore, the resistance against crack propagation, which is described by the fracture toughness $\left(K_{I C}\right)$, is an essential parameter for the characterization of advanced ceramics. There are different experimental methods for the measurement of $K_{I C}$. One of the preferred methods is the Single Edge V-Notched Beam (SEVNB) method due to its reliability and easy application. For unstable fracture the major limiting feature of the method is the presence of a finite notch (root) radius which can lead to an overestimation of the fracture toughness. Above a critical notch radius the effect is proportional to the square root of the radius. Damani et al. measured the fracture toughness of several ceramic materials with different notch radii and concluded 
that the appearance of the notch effect is unavoidable and in order to obtain consistent results the notch radius should be below a critical value $[1,2]$. Moreover, the results of Damani et al. for a given material with a constant prescribed notch radius showed a scattering of fracture toughness values. There are several other published works on this topic [3-7]. For unstable fracture several measurements have to be performed in order to estimate the influence of the notch radius on fracture toughness. In this paper a special method is used, which enables stable crack growth. By measuring a single specimen it is possible to separate the effect of the finite notch radius and, thus, calculate the true fracture toughness. The performed tests have also shown that the optical determination of the crack tip via light microscope can be an additional error source. Using zirconia-toughened alumina (ZTA) a tiny region of the crack just at the tip cannot be seen with the microscope, because the crack opening is below the optical resolution. It means that the observed crack length is too small by a constant value, more or less independently from the crack length. This has to be taken into account when calculating the fracture toughness.

First of all, the used equations for calculating the fracture toughness during stable crack growth and the effect of the finite notch root radius on the fracture toughness will be given briefly. Next, the experimental procedure including preparation of specimens and the experimental setup will be introduced and afterwards two measurements with ZTA will be presented and discussed.

\section{Theoretical Background}

\subsection{SEVNB}

One of the commonly used experimental methods for the measurement of $K_{I C}$ is the 4-pointbending test, in particular the SEVNB method (see Fig. 1). Stable crack propagation can be achieved through bending of the specimen with a displacement controlled device. In order to avoid unstable failure of the specimen this mechanical setup has to be very stiff. 

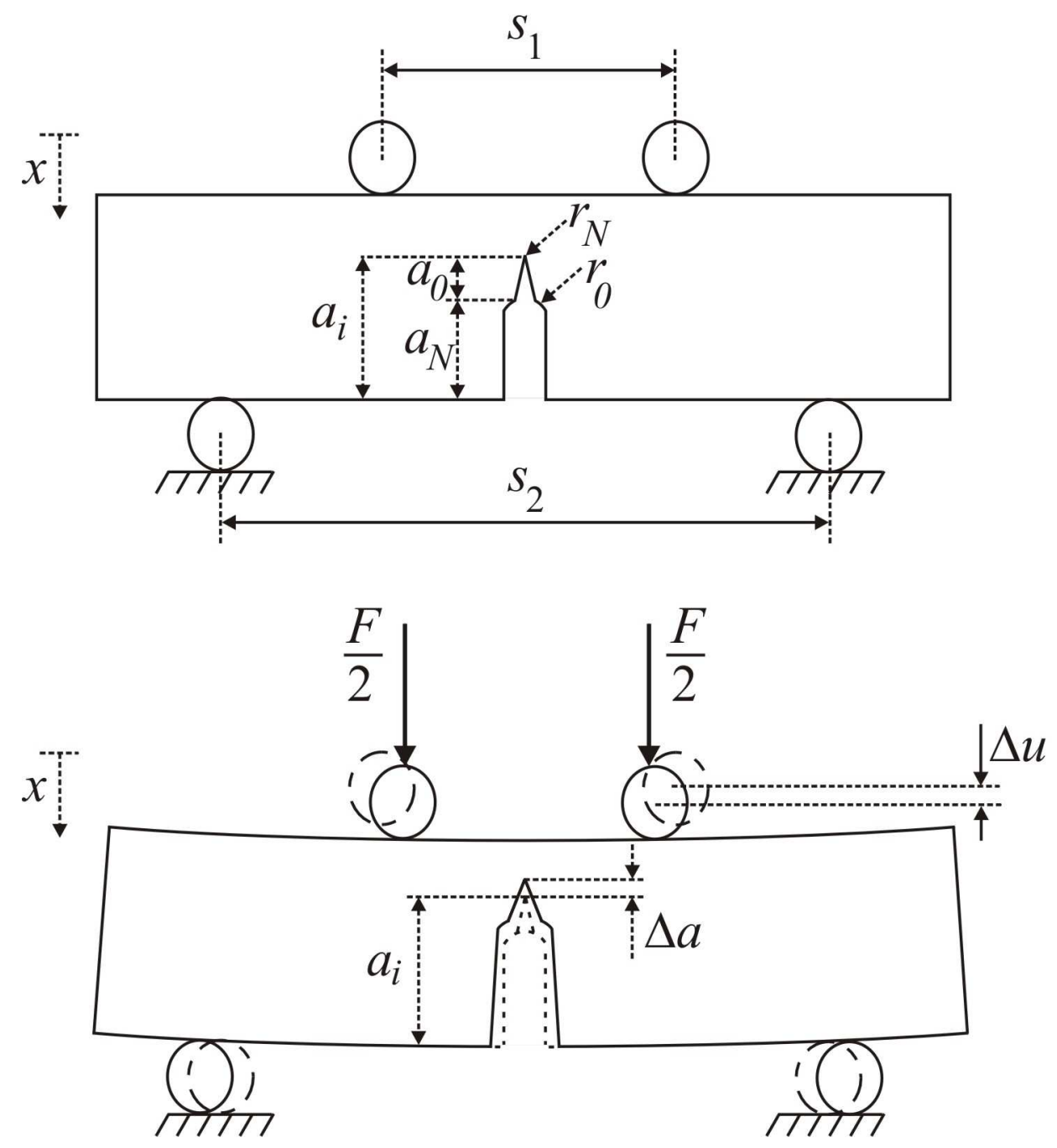

Figure 1: Schematic 4-point-bending setup, a) without and b) with external mechanical load.

Immediately after each crack propagation, the specimen is partially unloaded, so that the crack propagation $(\Delta a)$, the corresponding applied force $(F)$, and displacement $(\Delta u)$ can be recorded. Using the measured crack length $a=a_{i}+\Delta a=a_{0}+a_{N}+\Delta a$ (see Fig. 1) and the applied force the fracture toughness was calculated for each crack length by [8]:

$$
K_{I C}=\frac{3 F \Delta s \sqrt{\alpha} \Gamma(\alpha)}{2 b h^{3 / 2}(1-\alpha)^{3 / 2}}
$$

with $\quad \Gamma(\alpha)=1,1215 \sqrt{\pi}\left[\frac{5}{8}-\frac{5}{12} \alpha+\frac{1}{8} \alpha^{2}+5 \alpha^{2}(1-\alpha)^{6}+\frac{3}{8} \exp \left(-6,1342 \frac{\alpha}{(1-\alpha)}\right)\right]$,

$$
\alpha=a / h \quad \text { and } \quad \Delta s=s_{2}-s_{1}
$$


where $s_{1}$ is the inner and $s_{2}$ the outer distance of the support rollers, $b$ the width and $h$ the height of the specimen.

A typical force-displacement curve of a SENVB test with stable crack propagation is represented in Figure 2 (solid curve). A material with constant fracture toughness and no Rcurve is assumed. Here, crack propagation starts at the maximum attained force $F_{\max }$. Beyond this point, due to crack propagation, the applied force, required to extend the crack, decreases with decreasing sample stiffness. The thinner solid lines (notch lengths $a_{2}, a_{3}$ ) in Figure 2 indicate the loading of samples with larger initial notch lengths. It is $a_{1}<a_{2}<a_{3}$.

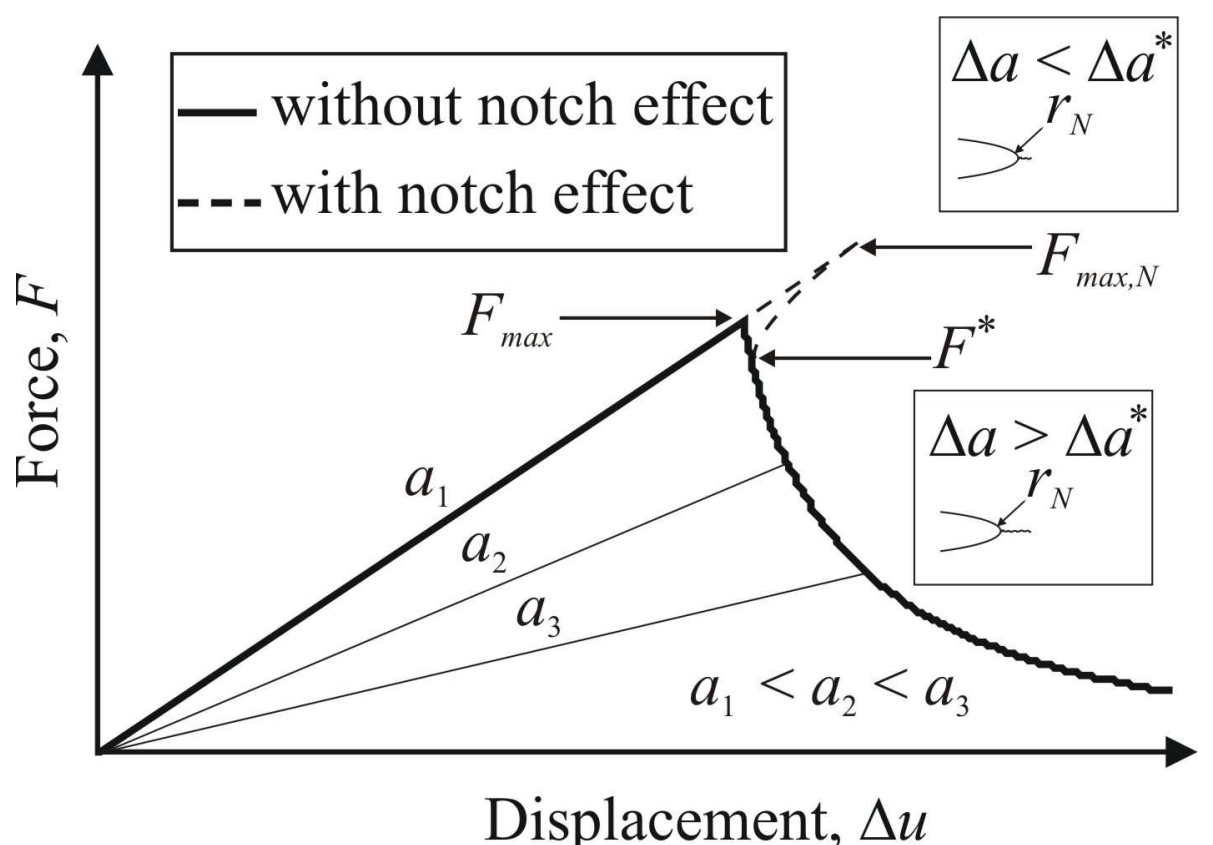

Figure 2: Schematic load-displacement curve for initial loading and stable crack growth with and without the influence of a finite notch root radius.

\subsection{Notch Effect}

With the conventional preparation of SEVNB-specimens it is not possible to obtain atomically sharp notches. Therefore, the influence of a finite notch radius $r_{N}$ has to be considered. Above a critical value of $r_{N}$ the measured nominal fracture toughness is higher than the true value. The amount of the overestimation depends on $r_{N}$ and the starting defect [1]. If the crack has propagated by more than $\Delta a^{*}=1.5 r_{N}$, the notch radius does not have any more significant influence on the fracture toughness [9].

The presence of a finite notch radius changes the shape of the force displacement curve as shown by a dashed line in Figure 2 . In this case the crack initiates at a nominal force $F_{\max , N}$, 
which is higher than $F_{\max }$. The indicated force $F^{*}$ is the point, where the crack extension is higher or equal to $\Delta a^{*}$ and consequently the influence of the notch effect disappears.

It is very difficult to estimate the exact magnitude of the gap $\Delta F=F_{\max , N}-F_{\max }$. First of all it cannot be absolutely ensured that $r_{N}$ is constant through the thickness of the sample. Moreover $a_{i}$ may not be the relevant crack length due to the occurrence of undetectable micro cracks, introduced at the crack tip during sample preparation. This uncertainty, when measuring $a_{i}$ and $r_{N}$, is a possible error source for the determination of the fracture toughness. A measurement with controlled crack growth helps to determine the influence of the notch radius and thus to get the true fracture toughness. Especially for materials with very strong rising R-curves like $\mathrm{Si}_{3} \mathrm{~N}_{4}$, the notch effect has to be taken into account in order to determine the correct R-curve [10-12].

\section{Experimental Procedure}

\subsection{Preparation of Specimens}

One long side of the ZTA bending bars $\left(\right.$ BIOLOX $^{\circledR}$ delta, CeramTec AG) with dimensions of about $3 \times 4 \times 25 \mathrm{~mm}^{3}$ (was polished in order to enable microscopic observation of the growing crack. A notch with length $a_{0}$ was introduced with a diamond saw blade of $120 \mu \mathrm{m}$ thickness and lateron sharpened with the razor blade method [13]. It is of importance that $a_{0}>1.5 r_{0}$ to avoid the effect of the finite notch radius $r_{0}$ induced by the saw blade.

Pilot tests had shown that stable crack growth in the brittle ZTA material was realizable with an initial crack length of at least $3.2 \mathrm{~mm}$. Two specimens were prepared with $a_{i 1}=3.217 \mathrm{~mm}$ and $a_{i 2}=3.305 \mathrm{~mm}$ and notch tip radii of $r_{N 1} \approx 3 \mu \mathrm{m}$ and $r_{N 2} \approx 5 \mu \mathrm{m}$. These values were determined with an optical microscope.

\subsection{Experimental setup}

The tests were performed in a very stiff displacement controlled four point bending device [14], where the support rollers have distances of $10 \mathrm{~mm}$ and $20 \mathrm{~mm}$ and a diameter of $5 \mathrm{~mm}$. Stable crack growth is conducted semi-automatically via a step motor and a computer aided control system. This has the advantage that all energetic mechanical processes in the sample, like a growing crack or micro cracking, are detected even without visible crack advance on the sample surface. This technique, based on a force-displacement evaluation, will be described in detail in a forthcoming publication [15]. 


\section{Results}

Force displacement curves of two ZTA specimens, measured with stable crack growth (Fig. 3) exhibit a maximum force $F_{\max }$ and thereafter a drop to $F^{*}$. In both experiments the crack initiation became optically visible, when the force had decreased to $F_{0}$, which is about $70 \%$ of $F^{*}$. It should be remarked that the registration of data points without any visible crack advance is possible only because of the automatic control system.
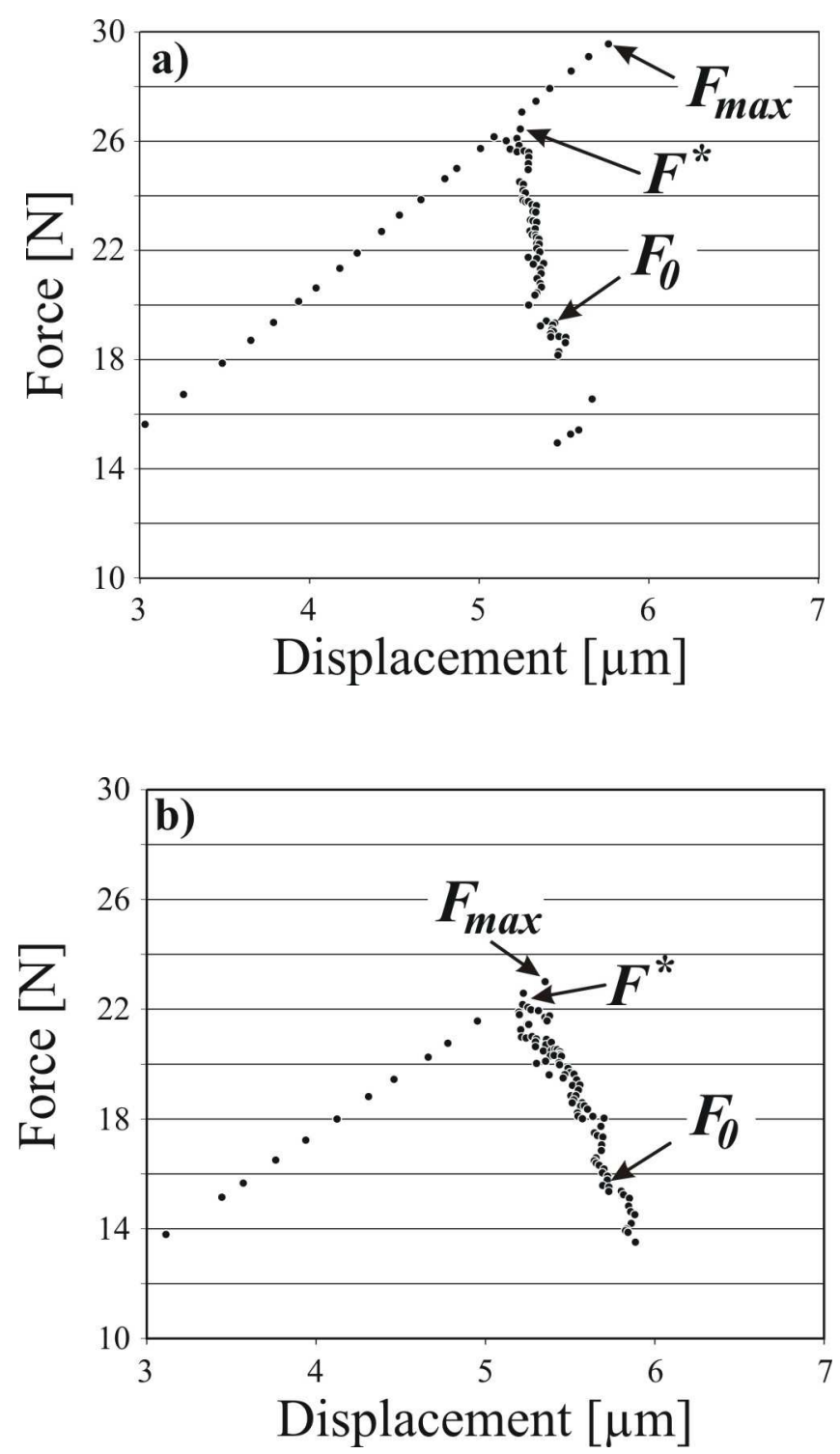

Figure 3: Load-displacement curve for stable crack advance in ZTA for a notch length of a) $3.217 \mathrm{~mm}$ (sample 1) and b) $3.305 \mathrm{~mm}$ (sample 2).

Constant fracture toughness levels were calculated by using equation 1 and plotted as dashed lines in Figure 4. The small shift of the theoretical lines when comparing Figure 4a) and 4b) are due to slightly different specimen geometry. The measured crack length and force values 
are given in this figure except for the force interval between $F^{*}$ and $F_{0}$, because in this interval no crack propagation could be seen on the sample surface. The point at $F_{0}$ indicates the last data point without visible crack propagation. Hence, in Figure 4 the forces $F_{\max }, F^{*}$ and $F_{0}$ were plotted with the assumption that these values correspond to the prepared initial crack length $a_{i}$.
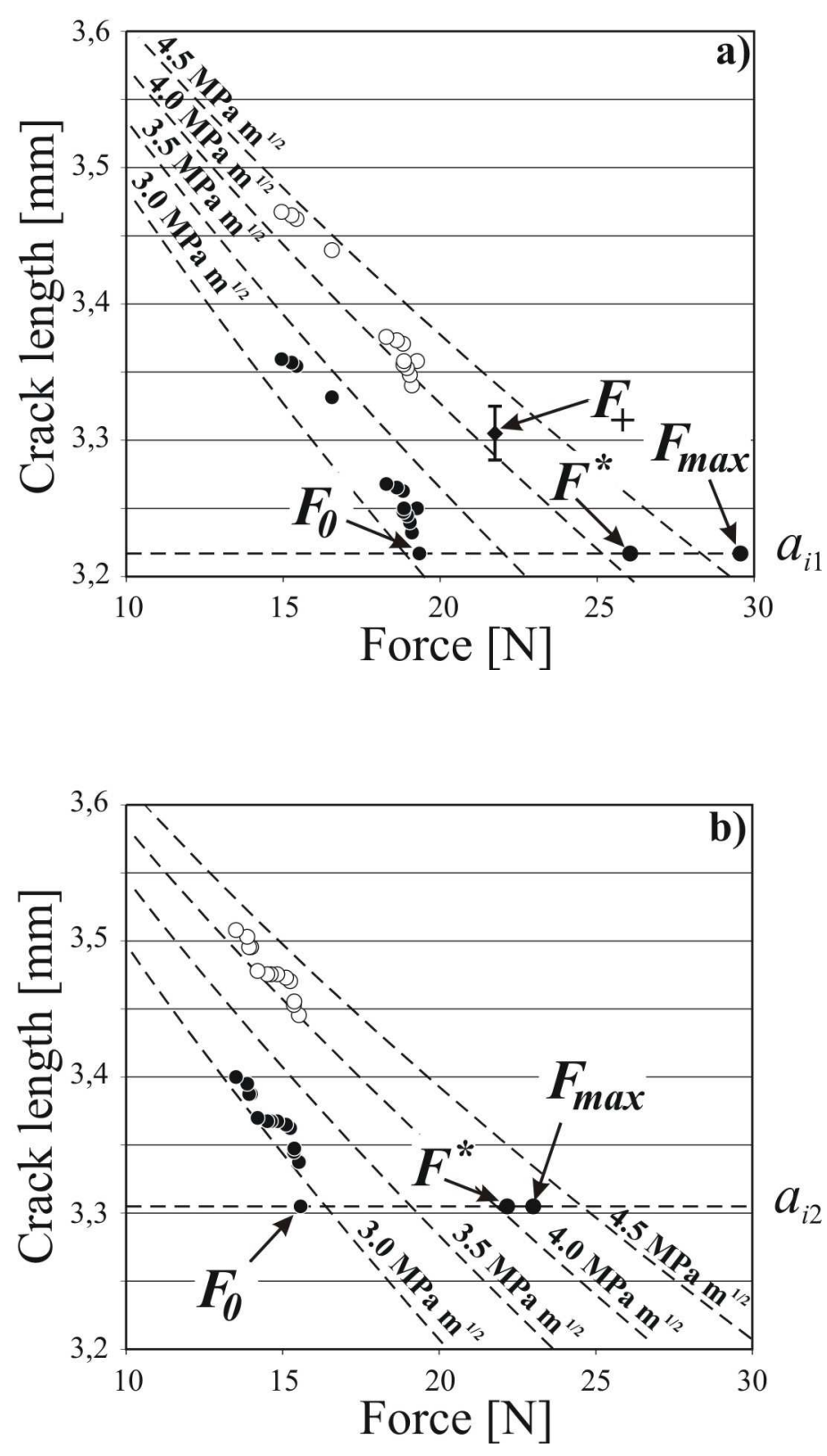

Figure 4: Crack length as a function of the mechanical load. The black circles correspond to the optically determined crack lengths, the open circles to crack lengths corrected by additional $108 \mu \mathrm{m}$ (see text further below). Initial notch length of a) $3.217 \mathrm{~mm}$ (sample 1) and b) $3.305 \mathrm{~mm}$ (sample 2). 
A closer comparison of the force displacements curves of the two specimens is given in Figure 5. Due to settling effects at small forces, the force-displacement curves do not behave linearly and thus the linear part at higher forces has to be extrapolated down to zero, where we place (arbitrarily) the origin.

The obtained peak in the force displacement curve is a typical indicator of a finite notch effect, since this leads to a higher nominal force required for crack initiation. It follows that crack propagation starts definitely at the attained maximum force $F_{\max }$. According to the trend of the curve, when the force drops to $F^{*}$, it can be concluded that the notch effect is not any more effective below $F^{*}$. The effect of the finite notch radius is only valid for approximately $\Delta a<1.5 r_{N}$ and the prepared notch radii are about $3 \mu \mathrm{m}$ and $5 \mu \mathrm{m}$ respectively. Because $1.5 r_{N} \ll a_{i}$ for the two samples $a_{i}+1.5 r_{N}$ is approximated by $a_{i}$ at $F^{*}$.

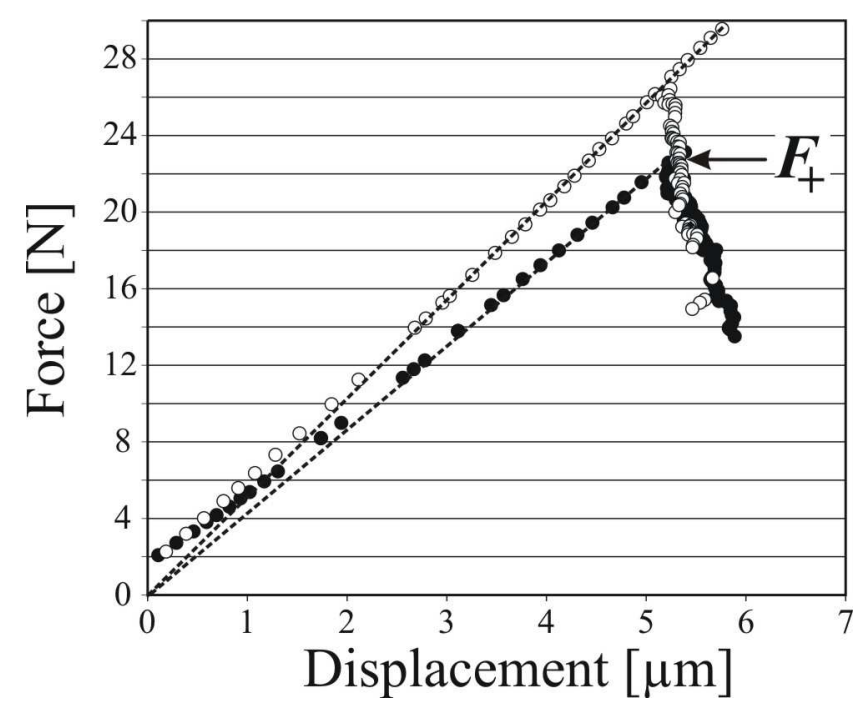

Figure 5: Both load-displacement curves of Fig. 3 in one diagram to visualize the region of the curves, corresponding to the $88 \mu \mathrm{m}$ difference of the notch lengths (sample 1: 0 , sample 2: •).

The fracture toughness using equation 1 with $F^{*}$ and $a_{i}$ is equal to $4.14 \mathrm{MPa} \sqrt{\mathrm{m}}$ (sample 1 ) and 4.06 $\mathrm{MPa} \sqrt{\mathrm{m}}$ (sample 2). For the data points below $F_{0}$, the fracture toughness is equal to $(3.23 \pm 0.06) \mathrm{MPa} \sqrt{\mathrm{m}}$ (sample 1) and $(3.11 \pm 0.05) \mathrm{MPa} \sqrt{\mathrm{m}}$ (sample 2) obtained with the optically measured crack lengths. The crack initiation starts at $F_{\max }$ and so the crack must have been grown between the forces $F^{*}$ and $F_{0}$. As a result, the optically determined crack lengths do not correspond to the relevant crack tip positions for calculating the fracture 
toughness. In fact, the crack length is too small. Therefore, the optically measured crack lengths have to be corrected by a constant offset in a systematical and reproducible way.

In Figure 5 the point, where both force displacement curves intersect, is at $F=F_{+}$. Assuming that the slope of the curve is changing only due to different crack lengths, it can be concluded that the initial crack in sample 1 propagates until this intersection point. It is suggested here that the amount of crack propagation should be roughly equal to the difference between the initial crack (notch) lengths of the two specimens, which is $\Delta a_{i}=a_{i 2}-a_{i 1}=88 \mu \mathrm{m}$.

Therefore the crack extension of $88 \mu \mathrm{m}$ should be added to the initial crack length $a_{i 1}$ for this particular force $F_{+}$. This gives a fracture toughness of $4.14 \mathrm{MPa} \sqrt{\mathrm{m}}$, as shown in Figure 4 . The error bar results from an appropriately chosen offset uncertainty of $( \pm 20) \mu \mathrm{m}$. This value matches very well with the fracture toughness belonging to $F^{*}$ (for both samples), implying that we already reached the plateau value. Since $F_{0}$, where the crack becomes visible, is smaller than $F_{+}$the crack must have been propagated more than $\Delta a_{i}=88 \mu \mathrm{m}$. The actual offset value can be obtained by shifting the optically determined data points to the fracture toughness belonging to $F_{+}$, which is achieved by a crack extension offset of $\Delta a_{\text {off }}=108 \mu \mathrm{m}$. This fits all optically measured data points to the fracture toughness corresponding to $F^{*}$ and $F_{+}$(Fig. 4 , symbol $\circ$ ) for both samples. Thus, the fracture toughness is $(4.14 \pm 0.14) \mathrm{MPa} \sqrt{\mathrm{m}}$ (sample 1) and $(4.15 \pm 0.08) \mathrm{MPa} \sqrt{\mathrm{m}}$ (sample 2), except for the maximum force $\left(F_{\max }\right)$ resulting from the finite notch radius.

\section{Discussion}

The constant offset of the crack length could have different technical and physical reasons. 1. Technically, the resolution limit of the light microscope restricts the optical determination of the crack tip. 2. After each crack advance the specimen is partially unloaded to stop the growing crack. This slight unloading might close the crack at the tip, so that it is not possible to exactly see the crack tip position in this region. 3. A physical reason for the optically measured decreased crack length can be a curved crack front inside the specimen so that the actual crack length inside is longer than on the observed surface. It might also be that the crack has not the same length on both sides. 4. In principle the creation of a process zone due to phase transition or other inelastic processes could lead to an additional displacement without crack advance but not to a force drop. 
For the tested material this leads to a correction of about $1 \mathrm{MPa} \sqrt{\mathrm{m}}$. The amount of more than $30 \%$ is quite high. Although the effect depends on the actual notch length, sample geometry, and the SEVNB method, the effect in principle can arise also for other experimental setups and should be considered.

Apart from that, it should be noted that the performed calculations using equation 1 are based on the ideale case of a straight crack front through the sample. In principle, this is a simplification, which is not taken into account here, but probably can be neglected.

From the load-displacement diagram in Figure 3 it follows that above a crack extension of $\Delta a^{*}=1.5 r_{N}$ the fracture toughness is quite constant and equal to the plateau value of the Rcurve. On the other hand, this does not need to be true below $\Delta a^{*}$. So, in principle a very short increasing R-curve might exist for the given material at the very first few micrometers. This effect would be superimposed by the notch effect and cannot be seperated. In any case, such an effect would not influence the results of the given paper.

\section{Summary}

The tested zirconia-toughened alumina has a fracture toughness of $4.1 \mathrm{MPa} \sqrt{\mathrm{m}}$ and after a crack extension of nearly $10 \mu \mathrm{m}$ the tested ZTA material does not exhibit any increasing Rcurve behavior. Because of the finit notch root radius bending experiments with unstable cracking would lead to an overestimation of the fracture toughness. Here, this effect could be clearly seen and quantified by testing a single specimen due to automatic crack growth control.

The results also point out that it is difficult to observe the actual crack tip position correctly. Different effects can cause that the optical determination of the crack length via light microscope yields values, which are too short by a constant offset leading to a contiuous underestimation of the fracture toughness. The reasons could be the limited resolution of the light microscope, the closing of the crack tip because of partial unloading and a curved crack front. With the given experimental setup it is possible to account for these effects and thus obtain the true fracture toughness. It is shown also that the effect is not negligibly small. Even if the phenomena, described in this paper, can be smaller for other experimental setups, we hope that the given results help to clarify the principle problems when performing fracture toughness measurements. 


\section{References}

[1] Damani, R. J., Gstrein, R. \& Danzer, R., Critical notch-root radius effect in SENB-S fracture toughness testing. J Eur Ceram Soc 1996, 16, 695-702.

[2] Damani, R.J., Schuster, Ch. \& Danzer, R., Polished Notch Modification of SENB-S Fracture Toughness Testing. J Eur Ceram Soc 1997, 17, 1685-1689.

[3] Bertolotti, R. L., Fracture Toughness of Polycrystalline $\mathrm{Al}_{2} \mathrm{O}_{3}$. J. Am. Ceram. Soc. 1973, 56-2, 107.

[4] Ando, K., Iwasa, M., Kim, B. A., Chu, M. C. \& Sato, S., Effect of crack length, notch root radius and grain size on fracture toughness of fine ceramics. Fatigue Fract. Engng. Mater. Struct. 1993, 16, 995-1006.

[5] Nishida, T., Hanaki, Y. \& Pezzotti, G., Effect of Notch-Root Radius on the Fracture Toughness of a Fine-Grained Alumina. J. Am. Ceram. Soc. 1994, 77, 606-608.

[6] Rocha, C. V. \& Costa, C. A., Effect of Notch-Root Radius on the Fracture Toughness of Composite $\mathrm{Si}_{3} \mathrm{~N}_{4}$ Ceramics. JMEPEG 2006, 15, 591-595.

[7] Fischer, H., Waindich, A. \& Telle, R., Influence of preparation of ceramic SEVNB specimens on fracture toughness testing results. Dental Materials 2007, 27, 618-622.

[8] Fett, T. \& Munz, D., Stress Intensity Factors and Weight Functions, Computational Mechanics Publications, Southampton, 1997, pp. 107-108.

[9] Fett, T., Influence of a notch root radius on fracture toughness. J Eur Ceram Soc 2005, 25, 543-547.

[10] Fett, T., Munz, M., Influence of narrow starter notches on the initial crack growth resistance curve of ceramics, Arch. Appl. Mech. 2006, 76, 667-679.

[11] Fett, T., Fünfschilling, S., Hoffmann, M.J., Oberacker, R., Jelitto, H. \& Schneider, G.A., R-curve Determination for the Initial Stage of Crack Extension in $\mathrm{Si}_{3} \mathrm{~N}_{4}$. J. Am. Ceram. Soc. 2008, 91, 3638-3642.

[12] Torres, Y., Bermejo, R., Llanes, L. \& Anglada, M., Influence of notch radius and Rcurve behaviour on the fracture toughness evaluation of WC-Co cemented carbides. Eng. Frac. Mech. 2008, 75, 4422-4430.

[13] Kübler, J., Fracture Toughness of Ceramics Using the SEVNB Method: From a Preliminary Study to a Standard Test Method. Fracture Resistance Testing of Monolithic and Composite Brittle Materials, ASTM STP 1409, J. A. Salem, M. G. 
Jenkins, and G. D. Quinn, eds., American Society for Testing and Materials, West Conshohocken, PA, 2002, 93-106.

[14] Jelitto, H., Felten, F., Swain, M. V., Balke, H. \& Schneider, G.A., Measurement of the Total Energy Release Rate for Cracks in PZT Under Combined Mechanical and Electrical Loading. Journal of Applied Mechanics 2007, 74, 1197-1211.

[15] Hackbarth, F., Jelitto, H., Özcoban, H. \& Schneider, G.A., Automatic R-curve measurement with different ceramic materials using a computer aided control system. (in preparation). 